\title{
A conservative approach to perineal Fournier's gangrene
}

\author{
Giulio Milanese ${ }^{1}$, Luigi Quaresima ${ }^{1}$, Marco Dellabella ${ }^{2}$, Alessandro Scalise ${ }^{3}$, \\ Giovanni Maria Di Benedetto ${ }^{3}$, Giovanni Muzzonigro ${ }^{1}$, Daniele Minardi ${ }^{1}$ \\ ${ }^{1}$ Department of Clinic and Specialistic Sciences, Urology, Polytechnic University of the Marches Region, \\ A.O. Ospedali Riuniti Ancona, Italy; \\ ${ }^{2}$ UOC Urologia, INRCA-IRCCS, Ancona, Italy; \\ ${ }^{3}$ Department of Experimental and Clinical Medicine, Plastic Surgery, Polytechnic University of the Marches Region, \\ A.O. Ospedali Riuniti Ancona, Italy.
}

\begin{abstract}
Summary Fournier's gangrene $(F G)$ is a disease involving necrosis of perineum and external genitalia; in $95 \%$ of cases it is possible to diagnose the Fournier's gangrene just by physical examination. The clinical presentation of $F G$ varies from an initial localized infection to large areas with necrotizing infection. The disease typically affect elderly men ( $6^{\circ}-7^{\circ}$ decade) with important systemic comorbidities; women are less frequently affected. Despite improvements in diagnosis and management, the mortality rate nowadays is between $20 \%$ and $43 \%$. The severity and mortality of the disease is dependent upon the general condition of the patient at presentation and upon the rate of spread of the infection. Treatment involves a multidisciplinary approach: intensive systematic management, broad-spectrum antibiotic therapy, early surgical debridement (wide abscission of necrotic tissues and surgical drainage of peritoneum, scrotum, penis, and inguinal areas), hyperbaric oxygen therapy; surgery can eventually be repeated if necessary; reconstructive surgery has an important role in the final treatment of the disease. The technical difficulties frequently encountered and the inability to make a complete removal of the necrotizing tissues at the time of surgery in some cases has led to the application of combined techniques, in view of the enhancement effect of specific advanced medications, targeted antibiotic therapy and hyperbaric medicine. We have considered 6 patients affected by Fournier's gangrene treated at our institution; all the patients received treatment with the help of plastic surgeons of the same institution. After debridement, all the patients were treated with advanced specific dressings consisting of plates and strips made of calcium alginate, hydrogels and polyurethane and twodimensional cavity foams. Reconstructive surgery was necessary in one case. Hyperbaric oxygen therapy (HBO) has been performed in all cases. The multidisciplinary approach, the combined use of HBO therapy and the adoption of advanced specific dressings, have made possible the complete healing of the lesions in a shorter period, avoiding further surgery in 5 out of 6 patients.
\end{abstract}

KEY WORDS: Fournier's gangrene; Hyperbaric oxygen therapy; Conservative management.

Submitted 23 February 2015; Accepted 15 March 2015

\section{INTRODUCTION}

At the end of XIX century Fournier described a disease characterized by an acute necrotizing process of cellulits and fasciitis of the penis and scrotum, rapidly evolving at high mortality and originally described as idiopathic gangrene of genitalia (1). Nowadays under the name of Fournier's gangrene (FG) we include disease which involve a necrosis of perineum and external genitalia; in 95\% of cases it is possible to diagnose the Fournier's Gangrene just by clinical presentation and examination. The necrotizing process commonly arises from an anorectal infection, or from an infection of the genitourinary tract or of the genital's dermis; less frequently it may develop as a consequence of acute leukemia, systematic erythematous lupus, Crohn's disease or HIV infection; FG can also be caused by iatrogenic and non-iatrogenic perineal trauma (2). It is also known that other systemic diseases, that can compromise the immune system, have a key role in the development of the necrotizing process (diabetes mellitus, obesity, chronic alcoholism, cirrhosis, immunosuppressive therapies, etc.).

The clinical presentation of FG varies from an initial localized infection to large areas with necrotizing extended infection. The disease typically affect elderly men $\left(6^{\circ}-\right.$ $7^{\circ}$ decade) with important systemic comorbidity; women are less frequently affected.

Despite the improvements in diagnosis and management, the mortality rate is nowadays between $20 \%$ and $43 \%$ (3-9). The severity and mortality of the disease is dependent upon the general condition of the patient at the presentation and upon the rate of spread of the infection. High hematic values of creatinine, bicarbonate, leukocytes and sodium represent negative independent prognostic factors. The spread of necrosis to the abdominal wall or even worse to the peritoneum means that there is no space for resolution of the situation by surgery only. For this reason the treatment involves a multidisciplinary approach: intensive systematic management, broad-spectrum antibiotic therapy, early surgical debridement (wide abscission of necrotic tissues and surgical drainage of peritoneum, scrotum, penis, and inguinal areas), hyperbaric oxygen ( $\mathrm{HBO}$ ) therapy; surgery can eventually be repeated if necessary; reconstructive sur- 
gery has an important role in the final treatment of the disease $(2,4)$. Surgical reconstructive techniques used for the treatment of FG are represented by simple free skin grafts to more complicated muscle flaps, myocutaneous (like the gracilis muscle) or fasciocutaneous flaps (as the anterolateral of the thigh or the "Singapore Sling"). These techniques are undoubtedly valid but present some drawbacks; in fact not all patients with FG are fit for surgery. The reconstruction with free skin grafts often brings drawbacks to the patients, mainly dimpling of the testicles and low plasticity of the new skin. The myocutaneous flaps are definitely the best choice but their use is limited in many cases by the complex surgical technique. The technical difficulties frequently encountered and the inability to make in some cases a complete removal of the necrotic tissues at the time of surgery has led to the application of combined techniques; in fact specific advanced medications, targeted antibiotic therapy and hyperbaric medicine have a combined effect that enhances each other.

\section{Patients And methods}

We have considered 6 patients affected by FG treated in the period April 2010-October 2012 at the Institute of Urology of the Polytechnic University of Marches Region, A.O. Ospedali Riuniti Ancona, Italy; all the patients received treatment with the help of the medical staff of the Institute of Plastic Surgery of the same Institution. We have evaluated the severity of the disease at the presentation using the Fournier's Gangrene Severity Index (FGSI) (3), taking into consideration the clinical and instrumental data of all six patients at the moment of hospitalization. Depending on the severity of the presentation and the clinical conditions, some patients underwent to emergency surgical debridement and decompression in the operating theatre (4 patients) or as an outpatient procedure (2 patients). As first treatment, all patients received empirical treatment with parenteral broad spectrum antibiotics, and subsequently targeted antibiotic therapy on the basis of the results of colture investigations; in addition, all the patients received several sessions of hyperbaric oxygen therapy (90 min at 2.5 ATA) (average number of sessions 10 , range $7-12$ ).

Lesions were then reviewed as bedsores and classified on the basis of NPUAP (National Pressure Ulcer Advisory
Panel, http://www.npuap.org/) classification. All patients were treated with specific advanced medications consisting of plates and strips made of calcium alginate, hydrogels and polyurethane and two-dimensional cavity foams. Reconstructive surgery was necessary in 50\% of cases.

\section{Results}

Mean patients age was 70.1 years. All patients are alive at a minimum follow-up of two years from the presentation. Table 1 summarizes the clinical and medical history of the patients. The patients were subjected to debridement with removal of necrotic tissues and consequent multiple loss of substance in the scrotal area $(n=4)$, buttock-perineal $(n=3)$, penile $(n=3)$, inguinaldeferential $(n=1)$ and suprapubic-inguinal $(n=1)$. In one patient at the time of the perineal debridement a wide necrotic lesion of the corpus spongiosum of bulbar urethra was detected. In two patients (2 men) debridement was performed as an outpatient procedure. A urethral catheter was placed in all the patients and also a suprapubic catheter in 4 patients. In all the patients the post-operative medications were performed on an outpatient basis using specialist advanced medications. In the first instance, calcium alginate dressings have been used until the lesion appeared well cleaned and granulating; then, when the healing process was good and there was not extensive loss of substance, hydrogel and polyurethane dressings were used $(n=4)$. In one case it was necessary the plastic reconstructive surgery using local flaps.

\section{Case reports}

\section{Case 1}

G.L., 71 years old woman. The patient was sent to our observation by the staff of a long-term care hospital where she was admitted two months earlier; she was having a poorly controlled insulin-dependent diabetes for 20 years and hypertension. At examination she had a large necrotic area in the inferior left gluteal, perineal and vulvar area, in communication between them.

The lesion involved the skin in its full-thickness, invading the underlying fascia with the exposure of the muscle. The FGSI was 8 . In emergency, surgery was made

Table 1.

Patients clinical and medical history.

\begin{tabular}{|c|c|c|c|c|c|c|c|c|c|c|c|c|}
\hline Pts & Age & Sex & Db & $\mathbf{R I}$ & I & Hp & Localization & Colture & Procedure & $2^{\text {nd }}$ surgery & HBO & Med. \\
\hline G.L. & 71 & $\mathrm{~F}$ & + & - & 8 & + & Gluteum-perineum-vulva & Pseud+candidae & Op theatre & no & 30 & 230 \\
\hline$\overline{\text { L.L. }}$ & 73 & $\bar{M}$ & + & - & 7 & - & Scrotum & Clostridium & Outpatient & no & 15 & 55 \\
\hline S.I. & 69 & $\mathrm{~F}$ & + & - & 8 & + & Gluteum-perineum-vulva & E.Coli+Morganella & Op theatre & yes & 30 & 240 \\
\hline$\overline{\text { G.I. }}$ & 65 & $\mathrm{M}$ & + & + & 13 & + & $\begin{array}{l}\text { Scrotum-penis-inguinal } \\
\text { and suprapubic region, urethra }\end{array}$ & Streptoc+Clostridium & Op theatre & no & 30 & 120 \\
\hline$\overline{P . P .}$ & 75 & M & + & - & 10 & + & Scrotum-perineum & Streptoc. beta emol. & Outpatient & no & 30 & 90 \\
\hline$\overline{\mathrm{N} . \mathrm{C}}$ & 68 & $\bar{M}$ & + & + & 12 & + & Scrotum-perineum & Proteus+E.Coli & Op theatre & no & 30 & 90 \\
\hline
\end{tabular}




\section{Figure 1.}

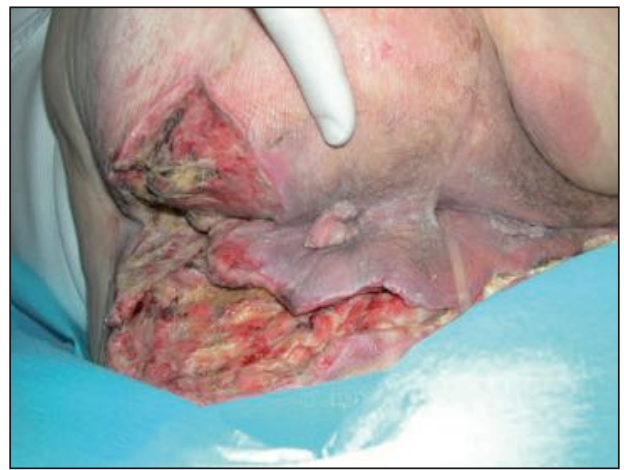

Figure 2.

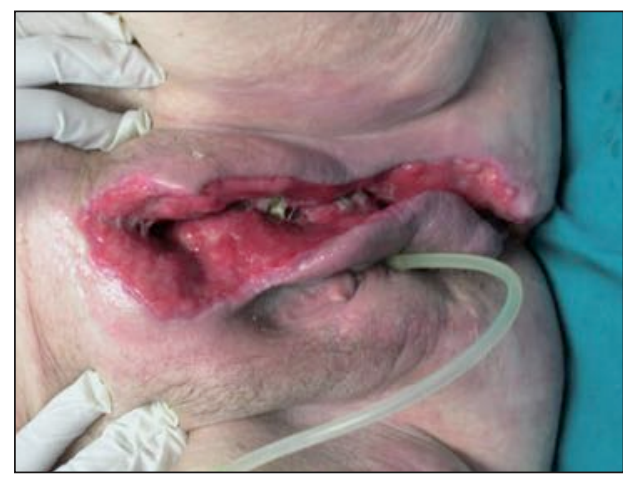

Figure 3.

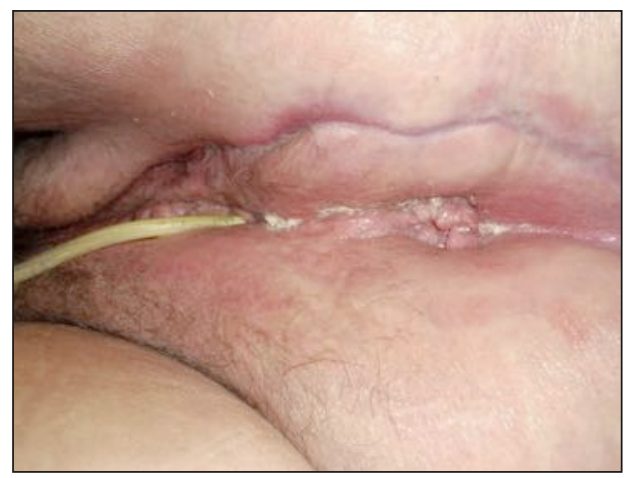

with exposure of the necrotic foci and removal of the damaged tissues (Figure 1). A swab colture of the wound has been obtained during surgery, and an empirical broad spectrum antibiotic therapy was started. The patient underwent to two cycles of 15 sessions each of hyperbaric therapy (90 min per session at 2.5 atmospheres - ATA) and to three weekly dressings with calcium alginate, with progressive improvement (Figure 2).

Intravenous Ciprofloxacin and Fluconazole were started in the 5th post-operative day as soon as the report of the swab showed the presence of Pseudomonas aeruginosa and Candida albicans. The wound healing to therapy was good, making unnecessary further surgery. The process of wound repair was completed in about 230 days (Figure 3).

\section{Case 2}

L.L., 73 years old man. The patient was sent to our clinic by his general practitioner (GP). The patient reported of having for about 2 months two fistulous lesions in the scrotum; at examination they resulted to be of $4 \mathrm{~cm}$ and $7 \mathrm{~cm}$ respectively (Figure 4). The patient had insulin-dependent diabetes, cardiac failure; at presentation he showed leukocytosis and fever $\left(38.5^{\circ} \mathrm{C}\right)$; the total FGSI was 7 . The patient was judged unfit for surgery, therefore we proceeded to debridement in an outpatient setting under local anaesthetic. A wound swab has been taken. Because of the secretions from the lesions, hydrogel dressing + polyurethane foams were used at alternate days (Figure 5); simultaneously, the patient underwent to 15 sessions of hyperbaric oxygen therapy (90 min per session 2.5 ATA). The swab showed the presence of microorganisms of the Clostridium strains, and appropriate antibiotic therapy was started. Wound healing was complete in 55 days without doing further surgery (Figure 6).

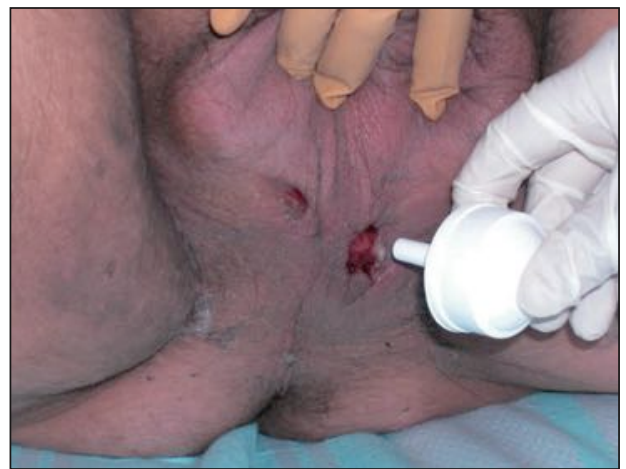

Figure 4.
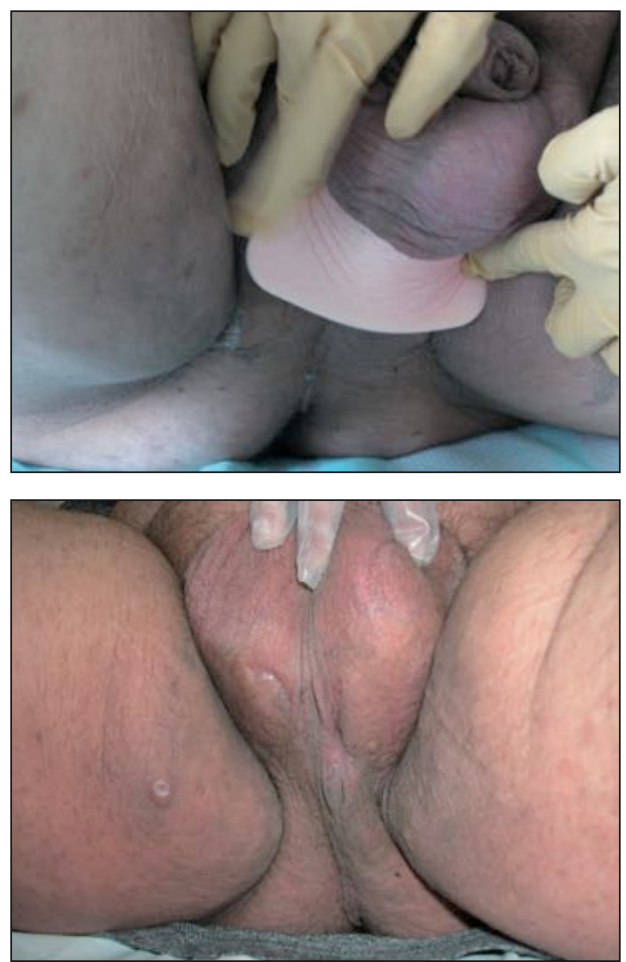

Figure 6.

\section{Case 3}

S.I., 69 years old woman. She was referred from a longterm care hospital, where she has been treated for about 1 year with antibiotic ointment and iodoform gauzes. The lesion involved the entire right gluteal, perineal and vulvar area, widely secreting and smelly.

The FGSI score was 8. At referral, the patient was taking insulin, digitalis, antihypertensives and broad-spectrum 
Figure 7.

Figure 8.
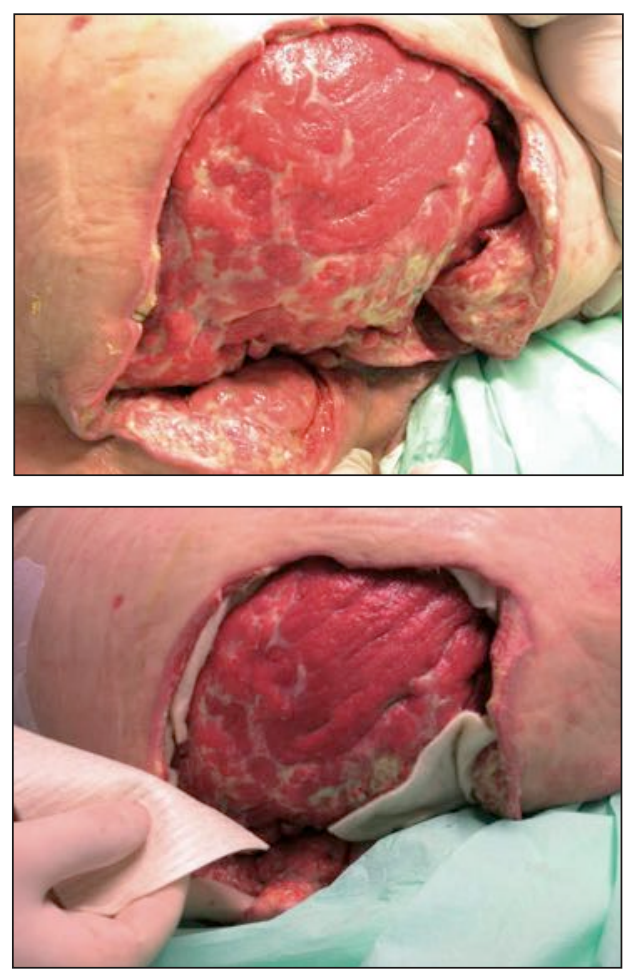

antibiotics. She underwent to emergency surgery, with opening of the infected regions (Figure 7); a wound swab was taken at the time of surgery; medications with calcium alginate were started (Figure 8) and also 2 cycles of hyperbaric therapy (90 min per session 2.5 ATA). The swab was found to grow E. coli and Morganella, and a specific therapy was started. Within five months there was improvement; but in consideration of the width of the lesions, it was decided to proceed to further surgery, and two fasciocutaneous flaps from the gluteal region were prepared, to cover the loss of residual substance; in the post-operative period there was a dehisce of a small part of the wound, treated conservatively; the ulcer was completely healed after about eight months.

\section{Case 4}

G.I., 65 year old man, referred from another hospital for pain and necrosis of genitalia, associated with leucorrhea from the urethra; the lesion started about 2 weeks before, and suddenly involved the scrotum, the penis and the inguinal region. The patient had a history of hypertension, treated with beta-blockers and calcium antagonists, renal failure (serum creatinine 2.5) and poorly controlled diabetes. The FGSI was 13. The huge presence of edema associated with a strong gaseous component with crackling of the subcuticular tissue indicated emergency surgery; it consisted of decompression and cleaning of infected foci, with removal of a large amount of scrotal tissue (testicles were not involved in the necrotic process) and multiple decompressive incisions of the penile area, and of the inguinal and suprapubic region in order to prevent the necrotic process to reach the abdominal wall; during surgery we could observe the involvement of the bulbar urethra, which appeared necrotic for $2 / 3$ of its circumference for $3 \mathrm{~cm}$ of length. The patient then received daily med- ications using advanced dressings based of calcium alginate and iodoform gauzes. From the $20^{\text {th }}$ post-operative day the patient started 2 cycles of hyperbaric oxygen therapy (90 min per session 2.5 ATA). The swab colture showed the presence of Streptococcus coagulase-negative and Clostridium, and a specific antibiotic therapy was started. With this conservative treatment, the wound had a quick repair, so reconstructive plastic surgery could be avoided. A complete healing was observed after about 120 days. The retrograde urethrography performed after 100 days from the operation showed the fully restoration of the urethra.

\section{Case 5}

P.P., 75 years old man. The patient was referred from a nearby mental health center because of the presence of two lesions, one in the lower part of the scrotum and another perianal, with an underlying communication, and contaminated by feces. In addition to the psychiatric disease, the patient had a poorly controlled insulindependent diabetes and arterial hypertension; the FGSI was 10 . The patient did not consent to surgery, therefore debridement was done as an outpatient procedure, using a scalpel, a Volkmann spoon and full washing with betadine solution. Hyperbaric oxygen therapy was started early (2 cycles of 15 each, 90 minutes per session to 2.5 ATA), associated with daily wound dressings with calcium alginate tape. Targeted antibiotic therapy against betahemolytic Streptococcus was started. After about 60 days the wound appeared granulating, and the alginate dressings were replaced it with hydrogel and polyurethane foam. The complete healing of the wound was observed in 90 days.

\section{Case 6}

N.C., 68 years old man. XX AA. The patient came overnight to the emergency of our hospital seriously ill and with high temperature $\left(39.2^{\circ} \mathrm{C}\right)$ in the last 3 days. A fistulous lesion starting from perineal area, secreting smelling pus and crackling, was observed. In the medical history, the patient had non-insulin dependent diabetes, mild renal impairment and hypertension treated with calcium antagonists (FGSI 12).

During emergency surgery, it was observed that the fistula reached the inguinal orifice and the spermatic cord. The patient started two cycles of 15 sessions each of OTI (90 min per session 2.5 ATA); daily wound medications with ringer lactate solution and covering with calcium alginate tape were performed.

Targeted antibiotic therapy was started on the basis of the swab colture, that revealed the presence of Proteus and Escherichia Coli. The quick healing of the lesion avoided further surgery, with complete resolution in about 90 days.

\section{Discussion}

The Fournier's syndrome is a serious disease for which early treatment is mandatory, aiming to expose the foci of infections and to decompress the abscesses as soon as possible. The cornerstone of treatment of patients affected by the FG is the early and radical surgical removal of the necrotic tissue, as shown by numerous studies (2-9). 
Nevertheless, sometimes for different reasons (patient unfit for surgery, refusal to surgery, etc ..) it is not possible to perform surgical treatment and alternative solutions need to be found out.

Hyperbaric oxygen (HBO) therapy is widely recognized as a valid therapeutic procedure, especially in cases with extended tissue necrosis $(2,10)$.

Furthermore, a conservative treatment based on the use of ancillary techniques (special medications) associated to HBO therapy can eliminate the need for repeated surgical debridement, and in selected cases, with low FGSI, it can represent an alternative way to reconstructive surgery. In some cases the width of the necrotic lesions would make delayed, if not extremely difficult, the reconstructive surgery; the use of specific medications has made possible a quicker tissue growth, speeding up the healing time and avoiding reconstructive surgery in some cases.

Laor et al. (3) developed a severity index for the FG in order to stratify risk groups in this complex patient population.

The Authors observed that a FGSI greater than 9 is a sensible and specific predictor of mortality for patients with FG, regardless of treatment.

In our series, 3 patients had a FGSI $<9$ and 3 a FGSI > 9; the use of specific advanced medications, in association with HBO therapy, has enabled rapid healing of large losses of substance and therefore avoided in 5 cases further reconstructive surgery.

Noteworthy, the re-epithelialization until complete healing of the wide urethral lesion was possible, without the need of reconstructive urethral surgery.

In conclusion the multidisciplinary approach, the combined use of HBO therapy and the adoption of advanced specific dressings, have made possible the complete healing of the lesions in a shorter period, avoiding further surgery.

\section{References}

1. Fournier JA. Gangrene foudroyante de la verge. Semin Med. 1883; $3: 345$.

2. Eke N. Fournier's gangrene: a review of 1726 cases. Br J Surg. 2000; 87:718-28.

3. Laor E, Palmer LS, Tolia BM, et al. Outcome prediction in patients with Fournier's gangrene. J Urol. 1995; 154:89.

4. Corcoran AT, Smaldone MC, Gibbons EP, et al. Validation of the Fournier's Gangrene Severity Index in a Large Contemporary Series. J Urol. 2008; 180:944-8.

5. Dahm P, Roland FH, Vaslef SN, et al. Outcome analysis in patients with primary necrotizing fasciitis of the male genitalia. Urology. 2000; 56:31.

6. Korkut M, Icoz G, Dayangac M, et al. Outcome analysis in patients with Fournier's gangrene: report of 45 cases. Dis Colon Rectum. 2003; 46:649.

7. Yeniyol CO, Suelozgen T, Arslan M, Ayder AR. Fournier's gangrene: experience with 25 patients and use of Fournier's gangrene severity index score. Urology. 2004; 64:218.

8. Tuncel A, Aydin O, Tekdogan U, et al. Fournier's gangrene: three years of experience with 20 patients and validity of the Fournier's Gangrene Severity Index Score. Eur Urol. 2006; 50:838.

9. Ersay A, Yilmaz G, Akgun Y, Celik Y. Factors affecting mortality of Fournier's gangrene: review of 70 patients. ANZ J Surg. 2007; 77:43.

10. Mindrup SR, Kealey GP, Fallon B. Hyperbaric oxygen for the treatment of Fournier's Gangrene. J Urol. 2005; 173:1975.

\section{Correspondence}

Daniele Minardi, MD (Corresponding Author)

d.minardi@univpm.it

Giulio Milanese, MD

Luigi Quaresima, MD

Giovanni Muzzonigro, MD

g.muzzonigro@univpm.it

Clinica Urologica, Università Politecnica delle Marche,

A.O. Ospedali Riuniti

Via Conca 71 - 60126 Ancona, Italy

Marco Dellabella, MD

UOC Urologia, INRCA, IRCCS, Ancona, Italy

Alessandro Scalise, MD

Giovanni Maria Di Benedetto, MD

Dipartimento di Medicina Sperimentale e Clinica, Chirurgia Plastica

Università Politecnica delle Marche, A.O. Ospedali Riuniti,

Ancona, Italy 